Article

\title{
Ang Negatibong Imahen ng Maynila sa Nobelang Inferno ni Dan Brown
}

F.P.A. Demeterio III

\begin{abstract}
This paper aims at clarifying and resolving the controversy that emerged in the Philippines after Dan Brown, in his novel Inferno, allegedly named Manila as a gate of hell. This article believes that there are important thoughts and lessons that can be gleaned from such controversy, not only for the residents of Manila but for all Filipinos as well. Hence, the first substantive section of this article evaluates in detail the actual text of Brown to determine which of its aspects are accurate or inaccurate, and which of its aspects are positive or negative. The second substantive section of this article explores the wider and deeper processes that contribute to the negative image of the said city. The controversy spawned by Brown's negative comment should be taken as an opportunity for the residents of Manila, and the Filipino people, to reflect on some festering problems that are eroding this once beautiful capital city.
\end{abstract}

Keywords: Brown, Inferno, Manila, Philippines

\section{Introduksiyon}

sang dating guro sa Ingles at Espanyol ang Amerikanong manunulat na si Dan Brown (ipinanganak: 1964), na naging kilala sa mundo ng 1 kulturang popular dahil sa kanyang mga nobelang thriller na Digital Fortress (1998), Angels and Demons (2000), Deception Point (2001), Da Vinci Code (2003), The Lost Symbol (2009), at Inferno (2013). Maliban sa Digital Fortress at Deception Point ang kanyang mga nobela ay kabilang sa kanyang serye tungkol sa propesor ng simbolohiya na si Robert Langdon. Dalawang nobela na sa seryeng ito ang naisapelikula, ang Angels and Demons at $D a$ Vinci Code, habang ang Inferno naman ay nasa proseso na ng pagsasapelikula. Noong unang lumabas ang nobelang Inferno napansin na ng ilang mambabasa at kritiko ang di kaaya-ayang deskripsiyon ni Brown tungkol sa Maynila. Ngunit dahil abala noon ang bansa sa eleksiyon, hindi masyado napagtuunang pansin ng nakararami ang naturang teksto.

(C) 2014 F.P.A. Demeterio III

http://www.kritike.org/journal/issue 14/demeterio june2014.pdf

ISSN 1908-7330

(cc) BY-NC 
Naging maiinit na paksa lamang ang nobelang ito noong matapos ang medyo mabilis na bilangan ng mga boto para sa naturang eleksiyon.

Ang kontrobersiyal na teksto ng nasabing nobela ay isang flash back sa buhay ni Dr. Sienna Brooks, ang babaeng bida, nang sumubok siyang pumunta sa Pilipinas, bago pa man nag-umpisang mag-aral ng medisina, bilang volunteer sa isang feeding program. Taliwas sa inaasahan niyang malaparaisong baybayin at kabayanan, ang mataong slum area ng Maynila ang bumulaga sa kanya.

Manila had six-hour traffic jams, suffocating pollution, and a horrifying sex trade, whose workers consisted primarily of young children, many of whom had been sold to pimps by parents who took solace in knowing that at least their children would be fed. ${ }^{1}$

Di naglaon, sa gitna ng kalunuslunus na sitwasyon sa Lungsod na ito bigla na lamang nawala sa sarili ang dati nang may depresyon na si Sienna at kumaripas nang walang direksiyon hanggang papunta sa isang squatter area. Sa baho, ingay at nakikinita niyang panganib sa pook na ito, inahalintulad niya ito sa gates of hell ng Divine Comedy ni Dante Alighieri (1265-1321), na nagsisilbi namang sub-text at inter-text ng nobelang Inferno. ${ }^{2}$ Sa nasabing squatter area natagpuan siya ng tatlong barumbado na agarang dumakma sa kanya para gahasain sa loob ng isang abandonadong kubo. Ang kaisa-isang saksi, isang matandang babae na nagluluto sa di kalayuan, ay wala man lamang reaksiyon sa desperadong sigaw ni Sienna.

They tore open her shirt, clawing at her soft skin. When she screamed, they stuffed her torn shirt so deep into her mouth that she thought she would choke. Then they flipped her onto her stomach, forcing her face into the putrid bed. ... Even as she prayed, she could hear the men laughing, taunting her as their filthy hands hauled her jeans down over her flailing legs. One of them climbed onto her back, sweaty and heavy, his perspiration dripping onto her skin. ${ }^{3}$

Noong inakala ni Sienna na talagang magagahasa na siya, bigla na lamang niyang narinig ang sigaw ng tatlong lalaki, naramdaman ang patak ng dugo sa kanyang likuran, at nakita ang kanilang mabilisang pagtakas.

${ }^{1}$ Dan Brown, Inferno: a Novel (New York: Doubleday, 2013), 459.

${ }^{2}$ Cf. ibid., 460.

${ }^{3}$ Ibid., 460. 
The old woman glared threateningly at the others, whipping her bloody knife through the air until the three men scampered off. Without a word, the old woman helped Sienna gather her clothes and get dressed. "Salamat," Sienna whispered tearfully. "Thank you." The old woman tapped her ear, indicating she was deaf. ${ }^{4}$

Matapos ang insidenteng iyon, nilisan ni Sienna ang Pilipinas na walang paalamam sa kanyang mga kapwa volunteer. Balik sa Britanya umatake muli ang kanyang depresyon kahit nilibang niya ang kanyang sarili sa pagsasanay sa martial arts. Doon nag-umpisa ang pagkalagas ng kanyang buhok. Kaya sa kabuuan ng nobela ay nakasuot ng mahabang blond wig si Sienna na lubos namang napapakinabangan ng naratibo para sa kanyang mabilisang pagbabalatkayo. Humantong si Sienna sa desisyong pumunta sa Estados Unidos para mag-aral ng medisina.

Marahil ang pinakadramatikong reaksiyon sa teksto tungkol sa Maynila ng nobelang Inferno ay ang sulat ni Atty. Francis Tolentino, Chairman ng Metropolitan Manila Development Authority, para kay Brown noong ika-23 ng Mayo 2013. Ang sentral na mensahe ng sulat na ito ay nilalaman ng ikalawa at ikatlo nitong talata:

While we are aware that yours is a work of fiction, we are greatly disappointed by your inaccurate portrayal of our beloved metropolis. We are displeased of how you have used Manila as a venue and source of a character's breakdown and trauma, much more her disillusionment in humanity.

More than your portrayal of it, Metro Manila is the center of Filipino spirit, faith and hope. Our faith in God binds us as a nation and we believe that Manila citizens are more than capable of exemplifying good character and compassion towards each other, something that your novel has failed to acknowledge. Truly, our place is an entry to heaven.

Ikinadidismaya ni Tolentino ang di umano paggamit ni Brown ng maling pagsasalarawan ng Maynila bilang pook kung saan nagkaroon ng

${ }^{4}$ Ibid., 461.

(c) 2014 F.P.A. Demeterio III http://www.kritike.org/journal/issue 14/demeterio june2014.pdf ISSN 1908-7330 
breakdown si Sienna at kung saan nakamtan ng babaeng bida ang reyalisasyon kung gaano ka walang pag-asa ang sangkatauhan. Iginiit ni Tolentino na makadios ang mga Manilenyo na may mabubuting kalooban, mga katangiang nakaligtaan di umano ni Brown na banggitin sa kanyang nobela. Para kay Tolentino ang Maynila ay di umano entrada patungong kalangitan.

Nilalayon ng papel na ito bigyang linaw at resolusyon ang kontrobersiyang umusbong mula sa teksto ni Brown, at himayin ang ilang hindi masyadong lantad na diskurso sa likod ng kanyang negatibong imahen ng Maynila. Paniniwala ng papel na ito na may mahahalagang kaisipan at aral na maidudulot ang kontrobersiyal na teksto ni Brown, hindi lamang para sa mga Manilenyo, kung hindi pati na rin sa lahat ng mga Pilipino. Kaya ang kasunod na seksiyon ng papel na ito ay sisipat sa teksto tungkol sa karanasan ni Sienna sa Maynila para alamin kung alin sa mga aspekto nito ang maituturing na tama o mali, o hindi kaya positibo o negatibo. Habang ang pangatlong seksiyon naman ay sisipat sa mga malawakan at malalimang diskurso na nasa likod ng negatibong imahen ng Maynila na maiintindihan lamang kapag ungkatin natin ang mga historikal at sosyo-politikal na sanhi nito, at kapag iuugnay ang naturang imahen sa kaubuang tema ng nabanggit nang nobela.

\section{Ang Teksto Tungkol sa Karanasan ni Sienna sa Maynila}

Mas madaling himayin ang teksto tungkol sa karanasan ni Sienna sa Maynila kapag alam natin ang balangkas ng nobelang Inferno. Ang matatawag nating pinakakontrabida sa kwentong ito ay ang Suwisong bilyonaryo at siyantistang si Bertrand Zobrist. Naniniwala si Zobrist sa ideolohiya ng transhumanism, na nagtuturo na dapat higitan nang higitan ng tao ang kanyang kasalukuyang katayuan sa pamamagitan ng pagpapabilis sa proseso ng ebolusyon gamit ang genetic engineering. Alam ni Zobrist na hindi pa handa ang siyensiya at teknolohiya para sa ganitong gawain, ngunit nangangamba siya na bago pa man makamtan ng siyensiya at teknolohiya ang kaalaman sa pagbabago sa mga katangian ng tao ay mangyayari na ang pagkakaroon ng sangkatauhan ng self-destruction dahil sa sobrang dami at kapal na ng populasyon sa mundo. Para pababain ang populasyon at mabigyan ang sangkatauhan ng sapat na panahon para maging perpekto ang siyensiya at teknolohiya ng genetics, gumawa si Zobrist ng biolohikal na bomba na itinanim niya sa isang pook pangturista sa Istanbul, Turkey. Dahil kumbinsido si Zobrist kung gaano kadakila at makasaysayan ang kanyang ginawa, bago siya nagpakamatay sa Florence, Italya, nag-iwan siya ng isang palatandaan (clue) para kay Dr. Elizabeth Sinskey, ang direktor ng World Health Organization, at isang naka-video na 
pahayag sa protektor ni Zobrist, na kilala lamang bilang "Provost." Ang parehong palatandaan at video clip ay ginawa gamit ang mga imahen at wika ng Divine Comedy ni Alighieri, partikular na ang unang parte nito na Inferno, na ginamit din ni Brown bilang pamagat ng buong nobela.

Para maintindihan ni Sinskey ang matalinghagang palatandaan na iniwan ni Zobrist, na sa kanyang palagay ay magtuturo sa kinaroroonan ng biolohikal na bomba, nagpatulong siya kay Langdon, na inilipad niya mula Boston, Massachusetts, patungong Florence. Para din bigyan ng proteksiyon ang kagustuhan ng pumanaw na kliyenteng si Zobrist, sinikap din ng Provost na hindi mahanap ni Langdon ang biolohikal na bomba. Ginamit ng Provost ang dating kasintahan ni Zobrist, na walang iba kung hindi si Sienna Brookes, para mahiwalay si Langdon sa grupo ni Sinskey. Hindi alam ng Provost na pati rin pala si Sienna ay gusto ding mahanap ang naitanim na bomba. Kaya, lumabas na tatlong paksiyon ang may interes para tukuyin ang kinaroroonon ng biolohikal na sandata laban sa lumulubong populasyon ng mundo: ang grupo ni Sinskey upang pigilan ang pagsabog ng bomba, ang grupo ng Provost upang protektahan ang kahilingan ni Zobrist, at si Sienna para sa isang hindi pa lantad na dahilan. Ang kani-kanilang pakipagunahan sa isa't isa, at sa paubos nang paubos na panahon ay nagdala sa kanila mula sa Florence patungong Venice, at hangang sa Istanbul. Sa bandang dulo ng nobela, lumilinaw na gusto din pala ni Sienna na pigilan ang pagsabog ng bomba ni Zobrist, kaya lumilinaw rin ang kanyang pagiging babaeng bida na katuwang ni Langdon sa naratibong ito.

Sa pagsipat natin sa teksto tungkol sa karanasan ni Sienna sa Maynila, tutugunan ng seksiyong ito ang mga sumusunod na katanungan: 1) inugnay nga ba ni Brown ang Maynila at ang gates of hell ni Alighieri?; 2) makatotohanan ba ang mga katangiang ginamit ni Brown sa kanyang pagsasalarawan sa Maynila?; 3) katanggap-tanggap ba ang paggamit ni Brown sa Maynila bilang konteksto at sanhi ng pagkakaroon ng breakdown, trauma at pagkadismaya ni Sienna sa sangkatauhan?; at 4) wala bang positibong aspekto ang kontrobersiyal na paggamit ni Brown sa Maynila?

\section{Maynila bilang Gates of Hell}

Ang ideya na tinawag di umano ni Brown ang Maynila na gates of hell ay nagmumula sa ilang Pilipinong nagbigay ng madamdaming reaksiyon sa naturang nobela. Ngunit, kapag babalikan natin ang kontrobersiyal na teksto, makikita natin na ang inahalintulad ni Sienna na gates of hell ay hindi ang Lungsod ng Maynila, kung hindi ang isang maliit na bahagi nito na isang hindi pinangalanang squatter area:

(c) 2014 F.P.A. Demeterio III

http://www.kritike.org/journal/issue 14/demeterio june2014.pdf

ISSN 1908-7330

$(\mathrm{cc}) \mathrm{BY}-\mathrm{NC}$ 
She cleared the tears and grime from her eyes and saw that she was standing in a kind of shantytown - a city made of pieces of corrugated metal and cardboard propped up and held together. All around her the wails of crying babies and the stench of human excrement hung in the air. ${ }^{5}$

Ang masamang amoy, ingay, at ang kanyang naramdamang parading na masamang mangyayari sa pook na iyon ang nagtulak kay Sienna na bansagan itong gates of hell. Alam na alam nating mga Manilenyo na may marami tayong ganitong klaseng pook sa lungsod: madumi, maingay at mapanganib. Sa halip na magalit tayo kay Brown, mas makatarungang magalit tayo sa kapabayaan ng ating pamunuan at sa sarili nating kawalan ng paki-alam.

\section{Iba pang mga Katangian ng Maynila ayon kay Brown}

Tutugunan ng sub-seksiyong ito ang katanungang "makatotohanan ba ang mga katangiang ginamit ni Brown sa kanyang pagsasalarawan ng Maynila" sa pamamagitan ng pagtugon ng mga mas tiyak na katanungan tungkol sa: sa Maynila bilang lungsod na may pinakamakapal na populasyon sa mundo; sa anim na oras na pagkakabuhol ng trapiko nito; sa masahol nitong polusyon; at sa talamak nitong prostitusyon.

Lungsod na may Pinakamakapal na Populasyon: sa isip ng maraming Manilenyo, at ng maraming Pilipino, sigurado silang hindi taglay ng Pilipinas ang titulong pinakamataong bansa sa mundo, o ang titulong bansa na may pinakamakapal na populasyon sa mundo. Kaya nakakagulat ang pagtawag ni Brown sa Maynila, ang kabisera ng bansa, na lungsod na may pinakamakapal na populasyon sa mundo. Hindi malayong ibinatay ni Brown ang kanyang nasulat sa sanaysay na "World's Densest Cities" ni Robert Malone na inilathala sa Forbes.Com noong ika-21 ng Disyembre 2006. Mula sa sanaysay na ito, makukuha natin ang kasunod na mga datos:

\begin{tabular}{|l|l|l|l|}
\hline \multicolumn{1}{|c|}{ Lungsod } & \multicolumn{1}{|c|}{ Bansa } & \multicolumn{1}{c|}{$\begin{array}{c}\text { Kapal ng Populasyon } \\
\left(\mathbf{T a o} / \mathbf{K m}^{2}\right)\end{array}$} & \multicolumn{1}{c|}{ Ranggo } \\
\hline Maynila & Pilipinas & 41,014 & $1^{\text {st }}$ \\
\hline Cairo & Ehipto & 36,618 & $2^{\text {nd }}$ \\
\hline Lagos & Nigeria & 20,000 & $3^{\text {rd }}$ \\
\hline Macau & Tsina & 16,521 & $4^{\text {th }}$ \\
\hline Seoul & Timog Korea & 16,391 & $5^{\text {th }}$ \\
\hline Dhaka & Bangladesh & 14,688 & $6^{\text {th }}$ \\
\hline Buenos Aires & Argentina & 13,680 & $7^{\text {th }}$ \\
\hline
\end{tabular}

${ }^{5}$ Ibid., 459-460,

(c) 2014 F.P.A. Demeterio III http://www.kritike.org/journal/issue 14/demeterio june2014.pdf ISSN 1908-7330 


\begin{tabular}{|l|l|l|l|}
\hline Jakarta & Indonesia & 11,360 & $8^{\text {th }}$ \\
\hline Kaohsiung/Taipei & Taiwan & 9,835 & $9^{\text {th }}$ \\
\hline Santo Domingo & Dominican Republic & 9,516 & $10^{\text {th }}$ \\
\hline
\end{tabular}

Tsart 1: Listahan ng mga Lungsod na may Pinakamakapal na Populasyon sa Mundo ayon sa Forbes.Com

Batay sa tsart na ito, lumalabas na matatag ang basehan ang pagtawag ni Brown sa Maynila bilang lungsod na may pinakamakapal na populasyon sa mundo. Ang mga Manilenyo at Pilipinong nagdududa kay Brown at kay Malone ay tiyak na maghahanap ng ibang datos na magpapasinungaling sa dalawang manunulat. Tiyak rin na ang mga diskumpiyadong taong ito ay hindi mahihirapan sa paghahanap, dahil sa sanaysay na "The Largest Cities in the World by Land Area, Population and Density," na inilathala sa Citymayors.Com, noong ika-2 ng Enero 2007, ay ganito naman ang datos na makukuha:

\begin{tabular}{|l|l|l|l|}
\hline \multicolumn{1}{|c|}{ Lungsod } & \multicolumn{1}{|c|}{ Bansa } & \multicolumn{1}{c|}{$\begin{array}{c}\text { Kapal ng Populasyon } \\
\left(\mathbf{T a o}_{\mathbf{K m}} \mathbf{K}^{\mathbf{2}}\right.\end{array}$} & \multicolumn{1}{c|}{ Ranggo } \\
\hline Mumbai & India & 29,650 & $1^{\text {st }}$ \\
\hline Kolkata & India & 23,900 & $2^{\text {nd }}$ \\
\hline Karachi & Pakistan & 18,900 & $3^{\text {rd }}$ \\
\hline Lagos & Nigeria & 18,150 & $4^{\text {th }}$ \\
\hline Shenzhen & Tsina & 17,150 & $5^{\text {th }}$ \\
\hline Seoul/Incheon & Timog Korea & 16,700 & $6^{\text {th }}$ \\
\hline Taipei & Taiwan & 15,200 & $7^{\text {th }}$ \\
\hline Chennai & India & 14,350 & $8^{\text {th }}$ \\
\hline Bogota & Colombia & 13,500 & $9^{\text {th }}$ \\
\hline Shanghai & Tsina & 13,400 & $10^{\text {th }}$ \\
\hline Lima & Peru & 11,750 & $11^{\text {th }}$ \\
\hline Beijing & Tsina & 11,500 & $12^{\text {th }}$ \\
\hline Delhi & India & 11,050 & $13^{\text {th }}$ \\
\hline Kinshasa & Congo & 10,650 & $14^{\text {th }}$ \\
\hline Manila & Pilipinas & 10,550 & $15^{\text {th }}$ \\
\hline Tehran & Iran & 10,550 & $16^{\text {th }}$ \\
\hline Jakarta & Indonesia & 10,500 & $17^{\text {th }}$ \\
\hline Tianjin & Tsina & 10,500 & $18^{\text {th }}$ \\
\hline Bangalore & India & 10,100 & $19^{\text {th }}$ \\
\hline Ho Chi Minh & Vietnam & 9,450 & $20^{\text {th }}$ \\
\hline
\end{tabular}

Tsart 2: Listahan ng mga Lungsod na may Pinakamakapal na Populasyon sa Mundo ayon sa Citymayors.Com

Malaki ang pagkakaiba ang listahan ng Forbes.Com, kung saan nangunguna ang Maynila na may kapal na 41,014 katao bawat kilometro kuwadrado, sa listahan ng Citymayors.Com, kung saan panglabinglima lamang ang Maynila na may kapal lamang na 10,550 katao bawat kilometro kuwadrado. Ngunit hindi tayo dapat magtataka kung alin sa dalawang listahang ito ang tama, dahil maaaring tama sila pareho. Kapag bubusisihin 
natin ang ibang detalye ng dalawang sanaysay, lalabas na ang pagkakaiba sa dalawang listahan ay naka-ugat sa magkakaibang "Maynila" na tinutukoy nila: ang Lungsod ng Maynila mismo sa sanaysay sa Forbes.Com, habang ang buong Metro Manila naman sa sanaysay sa Citymayors.Com. Batay sa impormasyong ito, maaari nang huminga ng mas maluwag ang mga mamamayan ng ibang bahagi ng Metro Manila, ngunit hindi ang mga Manilenyo.

Ang tanging konsuwelo ng mga taga Lungsod ng Maynila ay ang kaisipang baka hindi naman talaga ganoon ka kapal ang populasyon ng kanilang lungsod, dahil baka naman napalaki lang ang tala ng populasyon nito dahil sa mga transient na manggagawa at mag-aaral na sa Maynila nga pumapasok ngunit sa ibang lungsod ng Metro Manila, o hindi kaya sa mga karatig na probinsiya, naman umuuwi. Ngunit ang kawalan ng matatag na batayan ng pangkonsuwelong kaisipang ito ay may dala ring banta na baka din ang kasalukuyang tala ng populasyon ng lungsod ng Maynila ay hindi pa sumaklaw sa mga transient na manggagawa at mag-aaral. Ibig sabihin, maari ding lalong lumubo ang populasyon ng Lungsod sa oras at araw ng pasukan. Kaya mas makabubuti sa mga Manilenyo na tanggapin na lamang ang masaklap na katotohanang ang Maynila nga ang lungsod na may pinakamakapal na populasyon sa buong mundo at gamitin itong datos para sa isang masinsinan at pangmatagalang urban planning.

Anim na Oras na Pagkakabuhol ng Trapiko: sa pananaw ng mga Manilenyong tanggap na ang buhol-buhol na trapiko bilang bahagi ng buhay, nakakainis ang pahayag ni Brown na umaabot di umano ang pagkakabuhol-buhol na ito ng anim na oras. Ngunit kahit hindi man aabot sa anim na oras ang pang-araw-araw na pangyayaring ito, tiyak na hindi ito nalalayo sa sa anim na oras na binanggit ni Brown. Ilan sa mga pangunahing dahilan ng problemang ito ay ang lumulobo nating populasyon, dumadaming sasakyan, kahinaan sa ating sistema ng transportasyong pampubliko, kawalan ng regulasyon sa pag-phase-out ng mga lumang sasakyan, kawalan ng regulasyon sa mga sasakyang nakaparada sa mga kalye, kakulangan sa kakayahang gumawa ng mga bagong kalye, konsentrasyon ng mga pamantasan at mga tanggapan ng pamahalaan sa loob ng Lungsod, at ang lokasyon ng mga piyer, malalaking pamilihan at mall na nasa loob rin ng Lungsod.

Maaaring hindi kayang tugunan ng pamahalaan ang lahat nang sanhi na ito nang sabay-sabay. Ngunit kayang tugunan nito ang mga naturang sanhi ng paisa-isa. Sa halip na makipagdebate kay Brown kung talaga bang umaabot ng anim na oras ang pagkakabuhol-buhol ng trapiko sa Maynila, mas makabubuti para sa mga Manilenyo kapag intindihin na lamang nang mabuti ng ating pamunuan ang mga sanhi ng ating problema 
sa trapiko para alamin kung alin sa mga ito ang bibigyan nila ng priyoridad sa pagtugon sa susunod na tatlo o anim na taon.

Masahol na Polusyon: "Suffocating" ang pang-uri na ginamit ni Brown para sa polusyon ng Maynila, na ibig sabihin ay ang maruming hangin ang pangunahing aspekto ng kapaligiran ang tinumbok ng nobelista. Dahil nabanggit na natin ang padami nang padami na luma at bagong sasakyan na tumatakbo sa mga lansangan ng Lungsod at ang mahigi't kumulang anim nitong oras na pagkakabuhol ng trapiko, hindi mahirap tanggapin na nakakalunod nga ang polusyon sa hangin sa parehong Lungsod. Dapat pa nga tayong magtaka kung bakit hindi binanggit ni Brown ang polusyon na dulot ng basura at dumi na nakakalat sa ating mga kalye, estero at sa ipinagmamalaki nating Manila Bay. Sa sanaysay na "Twenty-Five Truths about Metro Manila," binanggit ng arkitekto at urban planner na si Paulo Alcazaren ang polusyon bilang pangalawang katangian ng metropolis kung saan parte ang Lungsod: "unless we convert to LPG and electric vehicles, build more sewage treatment plants, solve our solid-waste problem, clean our esteros and rivers... we will drown in chemical and biological excrement within a decade." 6

Ang polusyon sa hangin ay maaaring isisi natin sa ating pamunuan na hindi gumawa ng sapat na regulasyon sa paglimita sa bilang ng mga sasakyang pampribado, pag-phase-out sa mga lumang sasakyan, at hakbang para palakasin ang sistema ng pampublikong transportasyon. Ngunit ang polusyon na dulot ng basura ay dapat ding isisi sa ating kawalan ng pakialam habang nagtatapon ng kalat kung saan-saan. Mas madaling magalit kay Brown, o kay Claire Danes na noon ay nagpahayag din kung gaano ka dumi ang Maynila, kaysa magtanung sa sarili kung bakit ganito tayo ka walang disiplina at kung bakit ganito kataas ang ating tolerance sa kalat at maduming kapaligiran.

Talamak na Prostitusyon: "Horrifying" naman ang pang-uri na ginamit ni Brown para sa prostitusyong matatagpuan di umano sa Maynila, at sinabayan pa ito ng pahayag na ang karamihan daw sa mga sex worker dito ay mga minor de edad na ibinenta ng kanilang sariling magulang sa mga bugaw sa konsuelong maski papaano ay may makakain ang mga batang ito.7 Marihap pagdebatihan kung talaga bang "horrifying" ang prostitusyon sa Maynila, dahil relative ang pang-uring ito. Ibig sabihin, ang penomenon na "horrifying" para kay Brown ay maaaring normal at pangaraw-araw lamang sa ibang indibiduwal. Ang puwede nating busisihin dito

${ }^{6}$ Paulo Alcazaren, "Twety-Five Truths about Metro Manila," in Philippine Star (24

July 2011). Date Accessed: 02 November 2013, <http://www.philstar.com/sunday$\underline{\text { life/709025/25-truths-about-metro-manila> }}$.

7 See Brown, Inferno, 459.

(c) 2014 F.P.A. Demeterio III

http://www.kritike.org/journal/issue 14/demeterio june2014.pdf

ISSN 1908-7330

$(\mathrm{Cc}) \mathrm{BY}$-NC 
ay ang sinulat niya na karamihan daw sa mga sex worker sa Maynila ay di umano mga minor de edad, at ang mga ito raw ay di umano ibinenta ng kanilang sariling magulang sa mga bugaw. Mahirap paniwalaan ang mga pahayag na ito ni Brown, at wala tayong makikitang dokumento na magpapatunay nito.

Marahil ibinatay ni Brown ang kanyang eksaheradong pahayag sa mga balita tuwing may nagaganap na raid sa ilang kasa na kadalasan ay nagkukuwento ng pagkakaligtas ng madami-daming minor de edad. Ngunit, kahit na tanggapin na natin na karamihan nga sa mga sex worker ng mga na-raid na kasa na ito ay mga minor de edad, hindi ito nangangahulugang karamihan sa lahat ng sex worker sa Maynila ay mga minor de edad, dahil ang kasa ay isa lamang sa napakaraming sistema at kalakaran ng prostitusyon. Dagdag pa dito, ang karaniwang testimonya ng mga nailigtas na minor de edad ay nagsasalaysay ng kanilang maling akala na sa isang restaurant, tindahan, o kung ano pa mang lehitimong establisimento, sila ipapasok ng kanilang recruiter. Hindi pangkaraniwan ang testimonyang nagsasalaysay na ibinenta sila ng kanilang mga magulang sa may ari ng kasang kanilang hinantungan. Marahil ibinatay din ni Brown ang kanyang eksaheradong pahayag sa mga balita tungkol sa mga nahuhuling sindikato na may kinalaman sa child pornorgraphy sa internet. Pero ang kalakarang ito ay isang gawaing madalang at patago, na hindi kaagad masasakhihan ng kakarating pa lamang na Sienna.

Tama nang malaman natin na walang batayan ang pahayag ni Brown tungkol sa dami ng mga batang sex worker sa Maynila na ibinenta daw ng kanilang sariling mga magulang. Huwag na natin siyang sumbatan, dahil mas makabubuti para sa ating mga Manilenyo na tugunan na lamang ang hindi natin maitatangging problema ng prostituson, pati na ang prostituson ng mga minor de edad, at lalo na ang mga ugat at epekto ng mga problemang ito.

\section{Maynila bilang Tagpuan ng Krisis Sikolohikal ni Sienna}

Labis na ikinalulungkot ni Tolentino ang paggamit ni Brown sa Lungsod ng Maynila bilang tagpuan sa pagkakaroon ni Sienna ng breakdown, trauma at pagkakadismaya sa sangkatauhan. Ngunit, kapag babalikan natin ang nobela makikita nating hindi nakagugulat ang pagkakaroon ni Sienna ng breakdown. Ayon sa naratibo, dati nang may depression si Sienna bunsod sa kanyang pagiging child prodigy na nagpapabilis sa kanyang pag-aaral at nagbukod sa kanya sa kanya sanang mga kababata. Ang kanyang pagsali bilang volunteer sa mga gawaing pangkapwa ay paraan niya dapat para labanan ang kanyang depression. Nagkaroon siya muli ng breakdown sa Maynila dahil sa pagkakabigla niya sa

(c) 2014 F.P.A. Demeterio III

http://www.kritike.org/journal/issue 14/demeterio june2014.pdf

ISSN 1908-7330 
kulturang Manilenyo, lalo na siguro sa asta ng masang Manilenyo na sumusugod at nakikidumog tuwing mayroong relief operation. Alalahanin na lamang natin kung gaano ka dramatiko ang reaksiyon ng mga Manilenyo sa katatapus lamang na medical mission ng Iglesia ni Kristo noong ika-14 ng Oktubre 2013. Kung tutuusin, hindi ang Maynila ang sanhi ng breakdown ni Sienna, kung hindi ang kanyang sariling maselan na sikolohikal na katayuan dulot ng kanyang pambihirang talino at pagiging dating child prodigy.

Ang binanggit naman ni Tolentino na pagkakadismaya ni Sienna sa sangkatauhan ay maaaring nagmumula sa kasunod na mga pahayag:

Amid this chaos of child prostitution, panhandlers, pickpockets, and worse, Sienna found herself suddenly paralyzed. All around her, she could see humanity overrun by its primal instinct for survival. When they face desperation ... human beings become animals.

For Sienna, all the dark depression came flooding back. She had suddenly understood mankind for what it was - a species on the brink. ${ }^{8}$

Ang unang tatlong pahayag ni Brown ay eksaherado. Totoong laganap ang kahirapan sa Maynila, at totoong ding nangyayari ang prostitusyon at ibang krimen na kaakibat ng kahirapan, ngunit hindi totoong nag-aastang hayop na ang mga Manilenyo. Saglit lamang si Sienna sa Maynila, kaya hindi malayong hindi siya nagkaroon ng pagkakataong makilala kung gaano ka makatao ang mga Manilenyo, at kung gaano kainit sila makitungo sa mga panauhing dayuhan. Sa lingguwahe ng mga taong babad sa community involvement, si Sienna at kanyang mga kasama ay para lamang nag-parachute patungo sa komunidad na nagreresulta sa pagkakaculture shock ng parehong mga residente at panauhing dayuhan.

Ang huling pahayag ni Brown na "she had suddenly understood mankind for what it was-a species on the brink," ay dapat isasakonteksto sa kabuuang tema ng nobela: ang debate tungkol sa limitasyon ng mundo sa pagdala ng labis na populasyon. Gustong ipakita ni Brown na ang Maynila, bilang lungsod na may pinakamakapal na populasyon, ay may sobra-sobra nang dami ng tao na nasa bingit na ito ng pagkakaroon ng self-destruction. Ngunit may nakaligtaan si Brown. Una ang Lungsod ng Maynila ay may maliit lamang na aktuwal na bilang ng mga tao, na kapag ihahambing ito sa

${ }^{8}$ Ibid.

(c) 2014 F.P.A. Demeterio III

http://www.kritike.org/journal/issue 14/demeterio june2014.pdf

ISSN 1908-7330

(cc) BY-NC 
unang apat na lungsod sa mga listahan ng Forbes.Com at Citymayors.Com ay bababa ang Lungsod na ito sa bandang dulo:

\begin{tabular}{|l|l|l|l|}
\hline Lungsod & Bansa & Populasyon & Ranggo \\
\hline Cairo & Ehipto & $15,200,000$ & $1^{\text {st }}$ \\
\hline Mumbai & India & $14,350,000$ & $2^{\text {nd }}$ \\
\hline Lagos & Nigeria & $13,400,000$ & $3^{\text {rd }}$ \\
\hline Kolkata & India & $12,700,000$ & $4^{\text {th }}$ \\
\hline Karachi & Pakistan & $9,800,000$ & $5^{\text {th }}$ \\
\hline Shenzhen & Tsina & $8,000,000$ & $6^{\text {th }}$ \\
\hline Maynila & Pilipinas & $1,581,000$ & $7^{\text {th }}$ \\
\hline Macau & Tsina & 508,500 & $8^{\text {th }}$ \\
\hline
\end{tabular}

Tsart 3: Laki ng Populasyon ng mga Lungsod na Nangunguna sa mga Listahan ng Forbes.Com at Citymayors.Com

Pangalawa, ang Lungsod ng Maynila ay karugtong sa ibang parte ng Metro Manila, ang Pasay, Makati, Mandaluyong, San Juan, Quezon City, Caloocan at Navotas, na handang magsilbi na buffer zone kung sakali hindi na talaga kayang dalhin ng naturang Lungsod ang sobrang dami ng tao. Ibig sabihin, mas kapani-paniwala sana kung ang ginamit ni Brown na halimbawa ng lungsod na malapit na sa bingit ng pagkakaroon ng selfdestruction ay isang lungsod na nangunguna sa listahan ng mga mega-cities na may pinakamakapal na populasyon, katulad ng Cairo o Mumbai. Marahil nalilito si Brown sa pagkakaiba ng Metro Manila, na isa ngang megacity, at ng Lungsod ng Maynila, na hindi isang mega-city.

Ang trauma na binanggit ni Tolentino ay ang sikolohikal na epekto kay Sienna sa pagtangkang panggahasa sa kanya ng tatlong barumbado.

Sienna left the Philippines at once, without even saying good-bye to the other members of the group. She never once spoke of what had happened to her. She hoped that ignoring the incident would make it fade away, but it seemed only to make it worse. Months later, she was still haunted by night terrors, and she no longer felt safe anywhere. She took up martial arts, and despite quickly mastering the deadly skill of dim mak, she still felt at risk everywhere she went.

Her depression returned, surging tenfold, and eventually she stopped sleeping altogether. ${ }^{9}$

${ }^{9}$ Ibid., 461. 
Ang pagtangkang panggahasa, at panggahasa mismo, ay nangyayari kahit saan sa mundo, kaya hindi nakakasama sa Maynila ang ganitong negatibong kuwento. Sa katunayan, hindi natin mabibilang ang mga kuwentong naisulat at nailimbag na ng mga Filipino at Manilenyo tungkol sa panggahasa na nangyari sa Lungsod na ito na hindi man lamang natin pinuna noon. Halos ganito rin ang punto ng Filipino-American na manunulat na si Jessica Hagedorn (ipinangank: 1949) nang inihambing niya ang mga di kaaya-ayang imahen ng Maynila na binanggit ni Brown at ng kanyang sarili at mga kapwa manunulat sa librong Manila Noir: "What's the difference between what he's doing and what I'm doing. Why would mine be appreciated and his not?"10 Ngunit, dapat linawin pa rin natin na ang panggagahasa sa mga dayuhang babae ay hindi isang laganap na pangyayari sa Maynila. Ibang-iba ang pakikitungo ng mga Manilenyo, at ng mga Pilipino, sa mga dayuhan, mapababae man o lalaki. Nariyan palagi ang ating respeto at mataas na pagtingin sa nga dayuhan. Ang madalang na madalang na insidente ng panggagahasa ng dayuhang babae ay nangyayari lamang sa mga resort sa probinsiya. Kaya ang isang dayuhang babae na tatakbo sa isang slum area sa Maynila, o sa anumang parte ng Pilipinas, na tila wala sa sarili, ay siguradong makakatatanggap ng tulong at pagaasikaso mula sa mga Manilenyo, o sa mga Pilipino. Mapanganib man ang ilang pook ng Maynila, ngunit hindi realistiko ang eksena na isinulat ni Brown.

\section{llang Positibong Komento ni Brown tungkol sa Maynila at Pilipinas}

Ang teksto ni Brown tungkol sa karanasan ni Sienna sa Lungsod ng Maynila ay hindi puro negatibong komento laban sa nasabing Lungsod at pati na sa Pilipinas. Una, nariyan ang pahayag na: "Sienna imagined they were going to feed poor fishermen or farmers in the countryside, which she had read was a wonderland of geological beauty, with vibrant seabeds and dazzling plains" na nangangahulugang kinilala ni Brown ang tanyag nang kagandahan ng karagatan at kanayonan ng Pilipinas. ${ }^{11}$ Pangalawa, nariyan din ang eksena tungkol sa isang matandang babae na buong tapang humarap sa tatlong barumbado at nagligtas kay Sienna mula sa muntikang pagkagahasa. Pangatlo, ipinakita ng parehong parehong eksena ang tipikal na takot at paggalang ng mga Pilipino sa mga nakatatanda, mapababae man o lalaki, dahil madali sanang saktan ng tatlong barumbado ang matandang babae,

\footnotetext{
${ }^{10}$ Ruel de Vera, “Jessica Hagedorn on Dan Brown," in Inquirer Lifestyle (15 July 2013). Date Accessed: 01 November 2013, <http://lifestyle.inquirer.net/113993/jessica-hagedorn-ondan-brown-he-has-all-the-right-to-call-manila-gates-of-hell-but-he-cant-write>.

${ }^{11}$ Brown, Inferno, 459-460.

(C) 2014 F.P.A. Demeterio III http://www.kritike.org/journal/issue 14/demeterio june2014.pdf ISSN 1908-7330
}

(cc) BY-NC 
ngunit sa halip ay nagdesisyon silang tumakbo na lamang. Kaya, hindi ganoon kaangkop ang binitiwang salita ni Tolentino na: "Manila citizens are more than capable of exemplifying good character and compassion towards each other, something that your novel has failed to acknowledge."

Tutuldukan natin ang buong seksiyon na ito sa pamamagitan ng pagbanggit ng mahalagang realisasyon na ang kontrobersiyal na teksto ni Brown ay mistulang isang tela na hinabi gamit ang mga totoo at eksaheradong hibla. Dapat alam natin kung alin sa mga hiblang ito ang totoo at alin ang mga eksaherado. Alalahanin natin na ang eksaherasyon ay parte lamang ng diskurso ng naratibo, at hindi dapat igalit ng ninumang mambabasa. Dapat pa nga tayong magpapasalamat kay Brown, dahil binigyan niya tayo ng pagkakataong tumingin sa salamin, kahit pa man ang salaaming kanyang iniharap ay parang isang dispalinghadong salamin mula sa isang fun house, at magninilay kung alin sa mga nakikita natin tungkol sa ating sarili ang dapat nating baguhin at ayusin, at alin ang dapat nating panatiliin at pag-ibayuhin.

\section{Historikal at Sosyo-Politikal na Usapin sa Likod ng Negatibong Imahen ng Maynila}

Sa seksiyong ito, ituturing natin ang tela na hinabi ni Brown tungkol sa Maynila, kahit pa man gawa ito sa mga pinaghalong makatotohanan at eksaheradong hibla, bilang isang sintoma na nagpapahiwatig sa mga malalalim na usapin na dapat tugunan ng mga Manilenyo kung ayaw nating tuluyang magkakaroon ng self-destruction ang Lungsod. Nais ilantad ng papel na ito na ilan sa nangungunang historikal at sosyo-politikal na usapin sa likod ng negatibong imahen ng Maynila ay ang mga sumusunod: 1) ang problema sa populasyon, 2) ang pagiging parte ng Lungsod ng isang primate city, 3) ang Lungsod at ang puwersa ng entropy, 4) ang agwat sa pagitan ng mayayaman at mahihirap na taga Lungsod, 5) ang siklo at saklaw ng politikal na termino ng pamunuan at politisadong paraan ng paggastos, at 6) ang kakulangan sa kultural, pang-astetika at pangkapaligiran na edukasyon ng mga mamayan. Isa-isa nating talakayin ng mas malaliman ang mga usaping ito sa kasunod na mga sub-seksiyon.

\section{Problema sa Populasyon}

Ang pandaigdigang titulo ng Maynila bilang lungsod na may pinakamakapal na populasyon ay sapat na para puwersahin ang pamunuan ng Lungsod na pag-usapan na ang impact ng patuloy na pagdami ng mga Manilenyo. Kung hindi man kaya ng pamunuan na suwayin ang relihiyosong ideolohiya ng Simbahang Katoliko laban sa pagkakaroon ng 


\section{INFERNO NI DAN BROWN}

agresibong programa sa pamamahala ng populasyon (population management), dapat harapin ng pamunuan ang mas komplikadong implikasyon kung paano nito kupkupin ang karagdagang bilang ng mga isisilang na Manilenyo. Dapat maisip ng pamunuan na ang kanilang pagsunod sa dikta ng Simbahang Katoliko laban sa kontrasepsiyon ay may kapalit na napakalaki at napakabigat na responsibilidad tungkol sa pagbibigay ng makatao at desenteng pamumuhay sa padami nang padami na mga Manilenyo. Ang responsibilidad na ito ay lalo pang lalaki at bibigat kapat isaalang-alang natin ang katotohanang sa Lungsod na ito, at sa kabuuan ng Pilipinas, mas mabilis dumadami ang mga mahihirap kaysa mayayaman. Ang mga mahihirap ay may mas mataas na pangangailangan sa serbisyo publiko ng pamunuan at mas mababang ambag na buwis para sa kaban ng Lungsod.

Dapat lamang na ang Arsobispado ng Maynila ay makikisangkot na rin sa pamunuan ng Lungsod para sa paghahanap ng sustenableng paraan kong paano kargahin nang kargahin ng limitadong laki ng Lungsod ang dumadaming Manilenyo. Dahil sa pandaigdigang titulo ng Maynila bilang lungsod na may pinakamakapal na populasyon, hindi na makatarungan na ang papel ng lokal na simbahan ay hanggang sa paghaharang lamang sa anumang balak ng pamunuan na magkaroon ng programang pampopulasyon. Mas makatarungang harapin din ng lokal na simbahan ang mga praktikal na implikasyon ng kanyang iginigiit na teolohiya tungkol sa pamilya at seksuwalidad. Dapat na rin sigurong balikan ng mga teolohiko ng lokal na simbahan ang umuusbong na kontradiksiyon sa pagitan ng teolohiya ng pamilya at seksuwalidad, na nagbabawal sa agresibong pamamahala ng populasyon, at teolohiya ng paglika (creation), na nagtuturo naman na dapat ring pangangalagaan ang kapaligiran sa pamamagitan ng sustenableng pamumuhay.

\section{Maynila bilang Parte ng isang Primate City}

Kahit maging agresibo ang pamunuan ng Lungsod ng Maynila sa pagpapababa ng birth rate ng mga Manilenyo, hindi ito mangangahulugang magiging estabilisado na, o hindi kaya bababa na, ang populasyon ng Lungsod. Ito ay dahil parte ang Lungsod na ito ng Metro Manila na isang primate city. Ang konsepto ng primate city ay unang inilahad ng Amerikanong heograpo na si Mark Jefferson (1863-1949), at tumutukoy sa pinakamalaking lungsod ng isang bansa. Kadalasan, ang laki ng populasyon ng primate city ay mahigit doble sa laki ng populasyon ng

(c) 2014 F.P.A. Demeterio III

http://www.kritike.org/journal/issue 14/demeterio june2014.pdf

ISSN 1908-7330

(cc) BY-NC 
pangalawang lungsod ng parehong bansa. ${ }^{12}$ Batay sa laki ng populasyon ng primate city, karaniwang nagiging sentro ito ng politikal, pinansiyal, komersiyal, industriyal, kultural at pangserbisong mga aktibidad ng bansa. Ang mga aktibidad na ito ay nagreresulta naman sa pagkakaroon ng mga inprastraktura at oportunidad na sila namang humahatak sa mga indibiduwal mula sa ibang lungsod at pook ng parehong bansa. Nagmimistulang magnetikong puwersa ang inprastraktura at oportunidad na taglay ng isang primate city. Kaya lumalaki ang isang primate city hindi lamang sa pamamagitan ng birth rate ng kanyang mga residente kung hindi lalo na sa pamamagitan ng pagdagsa ng mga migrante. Ayon kay Jefferson, ang karaniwang ratio ng laki ng populasyon ng prime city, pangalawang lungsod at pangatlong lungsod ay 100: 30: $20 .{ }^{13}$

Batay sa deskripsiyon ni Jefferson, hindi maituturing na primate city ang Lungsod ng Maynila dahil ang populasyon nitong 1,581,000 ay hindi nalalayo sa populasyon ng ilang lungsod sa Pilipinas, katulad halimbawa ng Lungsod ng Kalookan na may populasyon na 1,489,040, o ng Lungsod ng Davao na may populasyon na 1,449,296, o ng Lungsod ng Quezon na may mas malaking populasyon na 2,761,720. Ang konsepto ng primate city ay magiging makabuluhan lamang sa konteksto ng Pilipinas kapag ang paguusapan ay ang kanyang mga metropolis, katulad ng Metro Manila, Metro Cebu at Metro Davao. Titingkad ang pagiging primate city ng Metro Manila kung ihahanay ang laki ng populasyon nito katabi sa laki ng populasyon ng Metro Cebu at Metro Davao:

\begin{tabular}{|l|l|l|l|}
\hline \multicolumn{1}{|c|}{ Metropolis } & \multicolumn{1}{|c|}{$\begin{array}{c}\text { Bilang ng Kasaping } \\
\text { Lungsod at Munisipyo }\end{array}$} & \multicolumn{1}{c|}{ Populasyon } & \multicolumn{1}{c|}{ Ranggo } \\
\hline Metro Manila & 17 & $11,855,975$ & $1^{\text {st }}$ \\
\hline Metro Cebu & 13 & $2,551,100$ & $2^{\text {nd }}$ \\
\hline Metro Davao & 7 & $2,262,518$ & $3^{\text {rd }}$ \\
\hline
\end{tabular}

Tsart 4: Laki ng Populasyon ng Unang Tatlong Metropolis sa Pilipinas

Batay sa tsart na ito, ang ratio ng laki ng populasyon ng Metro Manila, Metro Cebu at Metro Davao ay 100: 22: 19. Kung ihahambing ang ratio na ito sa karaniwang ratio na natuklasan ni Jefferson na 100: 30: 20, lalabas na lampas sa pangkaraniwan ang agwat ng laki ng populasyon ng Metro Manila kumpara sa laki ng populasyon ng Metro Cebu. Nagpapahiwatig ang ganitong agwat na sa konteksto ng Pilipinas isang dambuhalang primate city ang Metro Manila na may dambuhala ring magnetikong puwersa para humatak ng mga indibiduwal mula sa iba't ibang lungsod at pook ng bansa. Kaya sa gusto man o ayaw ng mga

12 See Mark Jefferson, "The Law of the Primate City," in Geographical Review, 29:2 (April 1939), 227.

${ }^{13} \mathrm{Ibid}$.

(c) 2014 F.P.A. Demeterio III http://www.kritike.org/journal/issue 14/demeterio june2014.pdf ISSN 1908-7330 
kasaping lungsod at munisipyo ng Metro Manila, kabilang na ang Lungsod ng Maynila, talagang lalaki at lalaki ang kani-kanilang populasyon dahil sa migrasyon ng mga Pilipino patungo sa kanilang dambuhalang primate city.

Hindi katulad sa problema ng mataas na birth rate na posibleng tugunan ng pamunuan ng Lungsod ng Maynila, ang usapin tungkol sa patuloy na paghatak ng Metro Manila ng karagdagang indibidwal ay isang bagay na lampas na sa kayahan ng pumunuan ng Lungsod ng Maynila pati na ng mga pamunuan ng mga kasaping lungsod at munisipyo ng Metro Manila. Kung naintindihan man ng pamunuan ng Lungsod ng Maynila at ng mga pamunuan ng mga kasaping lungsod at munisipyo ng Metro Manila ang dinamiko ng primate city, wala itong magawa maliban sa paglobby sa nasyunal na pamahalaan na labanan ang magnetikong puwersa ng Metro Manila sa pamamagitan ng intensibo at sistematikong pagpapalakas sa politikal, pinansiyal, komersiyal, industriyal, kultural at pangserbisong mga makinarya sa ibang metropolis, hindi lamang sa Metro Cebu at Metro Davao, kung hindi pati ang mga metropolitan area na nasa paligid ng Lungsod ng Baguio, Lungsod ng Dagupan, Lungsod ng Angeles, Lungsod Olongapo, Lungsod ng Batangas, Lungsod ng Naga, Lungsod ng Iloilo, Lungsod ng Bacolod, Lungsod ng Cagayan de Oro, at Lungsod ng Zamboanga.

\section{Ang Lungsod at ang Puwersa ng Entropy}

Kapag babasahin natin ang mga artikulo ni Alcazaren tungkol sa Maynila at urban planning, mararamdaman natin ang kanyang panghihinayang sa kumupas na ganda ng Lungsod (Tingnan halibawa sa Alcazaren, "Once upon a Fascinating Manila"). ${ }^{14}$ Gustong ipabatid ni Alcazaren sa ating mga Manilenyo na may panahon noon na napakaganda ang Maynila at nangunguna ang katayuan nito sa mga lungsod ng Asya. Ngunit, sa paglipas ng panahon, lumipas din ang ganda at katayuan ng Lungsod na tio. Ang puwersa ng entropy ay isang prinsipyo sa larangan ng pisika, partikular na sa disiplina ng thermodynamics, na nagsasalarawan sa likas na proseso ng disorganisasyon ng isang organisado ngunit aysoladong (isolated) bagay. Ang lungsod, bilang isang organisadong bagay, ay hindi ligtas sa puwersa ng entropy. Kaya para labanan ang puwersang ito, kailangan ng isang lungsod ang planadong pagpundar ng karagdagang lakas at materyales. Ngunit ang entropy ng lungsod ay sadyang mas komplikado kaysa entropy ng pisikal at walang buhay na bagay, at ang entropy ng nangyayari sa isang lungsod ay maaaring natatangi kung

\footnotetext{
14 See, for example, Paulo Alcazaren, “Once upon a Fascinating Manila," in Philippine Star (26 October 2013). Date Accessed: 02 November 2013, <http://www.philstar.com/modern-living/2013/10/26/1249339/once-upon-fascinating>.

(c) 2014 F.P.A. Demeterio III http://www.kritike.org/journal/issue 14/demeterio june2014.pdf ISSN 1908-7330
} 
ihahambing sa mga entropy na nangyayari sa ibang lungsod. Dapat maiintindihan ng pamunuan ng isang lungsod ang partikular na anyo ng entropy na nagbabanta sa kanilang lungsod.

Ang puwersa ng entropy na naghasik ng disorganisasyon sa Lungsod ng Maynila ay maaaring bunsod sa paglipat ng mga mayayamang Manilenyo papunta sa mga mas maluwag na subdibisyon na nag-umpisa pa noong natapos ang Ikalawang Pandaigdigang Digmaan, sa paglipat ng mga sentro ng komersiyo at pananalapi papunta sa Makati, Ortigas, Alabang at Bonifacio Global City, at sa pagdagsa ng mga mahirap na migrante mula sa mga probinsiya. Ang nakakalungkot isipin ay baka hindi man lamang naintindihan ng pamunuan ng Lungsod ng Maynila ang nakamamanghang puwersa ng entropy, kaya hindi rin napagtutuunang pansin ng pamunuang ito na kailangan palang intindihin ang anyo ng entropy na nagpapangit at sumira sa dating katayuan ng Lungsod, at lalong hindi nakapagplano ang parehong pamunuan kung paano labanan ang partikular na entropy na umiiral sa Maynila.

\section{Agwat sa Pagitan ng Mayayaman at Mahihirap na Lungsod}

Ang paglipat ng mga mayayamang Manilenyo patungo sa mga subdibisyon, at ang pagplipat ng mga sentro ng komersiyo at pananalapi patungo sa Makati, Ortigas, Alabang at Bonifacio Global City, ay may malaking implikasyon sa ekonomiya at katayuan ng Lungsod ng Maynila, dahil tinangay ng mga ito ang pera, kapital at oportunidad na magpapasigla sana sa kabuhayan ng Lungsod. Ang mahihirap na mayoriya na naiwan sa Lungsod ay may nakakubling puwersa na magtataboy sa mayayamang migrante at natitirang mayayaman, at humatak ng karagdagang mahihirap na migrante. Ayon sa teoryang broken windows, na unang iniladad ng Amerikanong sosyolihistang si James Wilson (1931- 2012) at Amerikanong kriminolohistang si George Kelling (ipinanganak: 1935), ang mga unang sintoma ng deteryorisasyon ng isang lungsod, o komunidad, ay nagsisilbing senyales para lumisan na ang mga naka-aangat na mamayan, na lalo namang magpapalala sa deteryorisasyon ng naturang lungsod, o komunidad. ${ }^{15}$ Ang deteryorisadong lungsod, o komunidad, ay makakahatak lamang ng mga mahihirap na migrante, dahil ayaw rin ng mga mayayamang migrante na tumagal sa ganitong uri ng na lungsod, o komunidad. Kaya hindi nakapagtataka na sa Lungsod ng Maynila lumalaki ang agwat sa pagitan ng mayayamang minoriya at mahihirap na mayoriya.

${ }^{15}$ James Wilson and George Kelling, "The Police and Neighborhood Safety: Broken Windows," in Manhattan Institute for Policy Research. Date Accessed: 02 November, $<$ http://www.manhattan-institute.org/pdf/_atlantic_monthly-broken_windows.pdf $>$. 


\section{INFERNO NI DAN BROWN}

Kapag dumadami ang mahihirap sa isang lungsod, hindi lamang pumapangit ang anyo nito, kung hindi mahihirapan din ang pamunuan nito sa pagtugun sa kanilang mga pangangailangan. Hindi katulad sa mga may kayang mamamayan na gumagastos sa kani-kanilang sariling edukasyunal at pangkalusugang pangangailangan, nakadepende ang sa pamahalaan ang mga mahihirap. Lalong pinapalala pa ito sa katotohanang kaunti lamang ang direktang naiaambag ng mga mahihirap na buwis para sa kaban ng lungsod. Dagdag pa dito, kapag dumadami ang mga mahihirap, tumataas din ang kriminalidad, prostitusyon, bagransiya (vagrancy) at bandalismo sa isang lungsod. Isipin na lamang natin bilang halimbawa ang mga nawawalang takip ng manhole, railings ng kalye, kawad ng kuryente at tansong estatwa sa mga pampublikong lugar na ikinalakal ng mga mahihirap sa mga junk shop. Dahil ang mga mahihirap ay kadalasang may mas mababa na edukasyunal na katayuan, madalas mababa din ang kanilang disiplina at urbanidad.

Noong tinatong tungkol sa kontrobersiyal na teksto ni Brown ang kultural na aktibista at tourism consultant ng Lungsod ng Maynila na si Carlos Celdran, sumagot siya na "talagang gate of hell ang Tondo at Ermita compared to Fort Bonifacio and Makati."16 Ang gustong ipahiwatig ni Celdran ay may pangit nga na aspekto ang Metro Manila, ngunit may mga planadong komunidad din ito na kayang makipagtagisan sa ganda at modernidad ng mga tanyag na lungsod sa Asya at kung saan man. Para kay Celdran, nagkataon lamang na ang pangit na aspekto ang nakita ni Brown. Ngunit, para sa mga Manilenyo problematiko ang pagiging planado, ganda at modernidad hindi lamang ng Makati at Bonifacio Global City, kung hindi pati na ang Ortigas at Alabang. Una, ang mga komunidad na ito ay kasama sa mga sanhi na tumangay sa pera, kapital at oportunidad na maaari sanang nagpasigla sa pamumuhay ng Lungsod ng Maynila. Pangalawa, hindi dapat tularan ng Lungsod ng Maynila ang mga komunidad na ito sa dahilang mga pribado itong proyekto na nakabatay sa ideolohiya ng kapitalismo na hindi naglalaan ng sapat na espasyo para sa mga mahihirap na Pilipino. Ang mas katanggap-tanggap na estratehiya na dapat dalumatin ng pamunuan ng Lungsod ng Maynila ay ang pagkakaroon ng modelo ng Lungsod kung saan may sapat na bilang at inklusibong espasyo ang mga mayayaman at mahihirap.

${ }^{16}$ Delon Porcalla, “Gates of Hell? Pinoys Know Better," in Philippine Star (25 May 2013). Date Accessed: 02 November 2013, <http://www.philstar.com/headlines/2013/05/25/ 946090/gates-hell-pinoys-know-better>.

(c) 2014 F.P.A. Demeterio III

http://www.kritike.org/journal/issue 14/demeterio june2014.pdf

ISSN 1908-7330

$(\mathrm{cc}) \mathrm{BY}-\mathrm{NC}$ 


\section{Siklo at Saklaw ng Politikal na Termino at Politisadong Paraan ng Paggastos}

Binanggit ni Alcazaren na may hindi bababa sa labing-dalawa nang master plan ang Maynila at Metro Manila na lahat ay hindi nasunod at nabaon na lamang sa limot. ${ }^{17}$ Kung bakit paulit-ulit itong nangyari sa kasaysayan ng Lungsod ay bunsod na rin sa hindi pagkakatugma sa pagitan ng laki at haba ng isang urban master plan, at sa pondo at haba ng isang termino ng isang pamunuan. Sa laki ng kinakailangang pera at panahon para maisakatuparan ang isang urban master plan, na ang epekto ay marahil mararamdaman lamang sa susunod pang ilang termino ng iba nang pamunuan, nawawalan ng lakas at gana ang mga halal na pinuno na itaguyod ang dambuhalang proyekto. Kalaban din ng isang urban master plan ang pangamba ng isang pamunuan na higupin ng proyektong ito ang lahat ng kanilang pera at panahon sa puntong wala na silang maipapakitang ibang buo na proyekto sa taumbayan. Kaya sa halip na itaguyod ang isang urban master plan, tumututok na lamang ang ang sinumang halal na pinuno sa mga maliit ngunit lantad na proyekto, kahit pa kadalasan ang mga ito ay panakip-butas lamang sa mga suliraning umusbong dulot na rin sa pagkasantabi ng parehong urban master plan. Kahit hindi na natin pag-uusapan ang korapsiyong naging kaugnay na sa usapin ng pampublikong paggastos, mahalagang makita natin na may problema mismo ang sistema ng politisadong pampublikong paggastos, dahil sadyang pumapabor ito sa mga mas mura, ngunit mas marami at mas lantad na proyekto, kaysa kaunti at hindi lantad ngunit istraktural na paggastos na may pangmatagalan at malaliman na epekto.

Hindi lamang ang temporal at pinansiyal na aspekto ng urban master plan ang problematiko, dahil problematiko rin ang heograpikal na hindi pagkakatugma sa pagitan ng lawak ng proyekto at pagkakahati-hati ng politikal na hurisdiksiyon ng Metro Manila. Inter-konektado ang pisikal na espasyo ng labingpitong lungsod at munisipyo na bumubuo ng Metro Manila, ngunit hindi inter-konektado ang mga pamunuan ng mga nasabing lungsod at munisipyo. ${ }^{18}$ Sa ganitong sitwasyon, mahirap magkaroon ng makatotohanang urban master plan na tanggap ng lahat ng labingpitong pamunuan. Dagdag pa dito, kahit na dahil sa isang milagro ay tunay na

\footnotetext{
17 See Paulo Alcazaren, "Ten Reasons Why Metro Manila is Losing its Soul," in Philippine Star (29 June 2013). Date Accessed: 02 November 2013, $<$ http://www.philstar.com/modern-living/2013/06/29/959355/ten-reasons-why-metro-manilalosing-its-soul>.

18 See Paulo Alcazaren, “Eleven Resolutions for Metro Manila," in Philippine Star (01 January 2011). Date Accessed: 02 November 2013, <http://www.philstar.com/modernliving/643880/11-resolutions-metro-manila>.
} 
nagkakasundo ang labingpitong pamunuan, may mga aspekto, tulad ng problema sa populasyon at labis na puwersa ng primate city, na lampas pa rin sa hurisdiksiyon ng buong Metro Manila at nasa hurisdiksiyon na ng nasyunal na pamunuan.

\section{Mga Edukasyunal na Pagkukulang}

Madalas ipinagyayabang ng mga Pilipino, kasama na ang mga Manilenyo, ang ating likas na talino at mataas na literacy rate, ngunit nakakalungkot isipin kung bakit ang mga ito ay hindi napapakinabangan sa pagsasaayos at pagpapaganda sa ating mga lungsod, komunidad at kapaligiran. Napansin noon ng pilosopong si Emerita Quito ang unibersalismo ng edukasyong sa Pilipinas, na sa pagiging abala sa humanistikong pagpapasakatuparan sa potensiyalidad ng bawat indibiduwal ay tila naubusan ng panahon sa pagtalakay sa mga pragmatikong usapin na nariyan sa ating sariling konteksto. ${ }^{19}$ Kaya napapanahon balikan ang mga pundasyunal na prinsipyo ng ating edukasyon at imumungkahi na bigyan ng sapat na diin ang pagsasapuso sa mga usapin tungkol sa ating kultura, pagpapahalaga sa kagandahan, at pangangalaga sa kapaligiran, kasama na ang mga dapat inaasahang gawain at tungkulin ng mga mamamayang nakatira sa isang urban na komunidad. Ilan lamang ito sa mga mga paksa na sang-ayon sa puna ni Quito ay dapat hahamon sa unibersalismo ng edukasyon sa Pilipinas. Kailangan ng mga Manilenyo, pati na ng mga Pilipinong nakatira sa ibang lungsod, at ng mga Pilipinong kalaunan ay luluwas din sa mga lungsod, ang pagkakaroon ng kamalayan na magtataguyod ng sustenableng pagsasaayos at pagpapagaganda sa ating mga lungsod. Ipinahiwatig ni Quito na ang unibersalismo ng edukasyon sa Pilipinas ay bumabagabag hindi lamang sa mga pampublikong paaralan, kung hindi pati na sa mga pampribadong institusyon. Ang kakulangan sa kamalayang kultural, astetikal at pangkapaligiran, pati na sa urbanidad, ng ating mga pinuno, na madalas ay mga produkto ng pampribadong edukasyon, ay patunay kung gaano kalaganap ang unibersalismong ito.

Kung mangyayari man ang ating paggamit sa edukasyon bilang sandata sa pagsasaayos at pagpapaganda sa ating mga lungsod, partikular na ang Lungsod ng Maynila, pangangalaga sa kapaligiran, at pagkakaroon ng urbanidad, dapat sasabayan ito ng mas midya at ng mga malinaw na ordinansa sa antas ng mga lungsod at bansa na tuloy-tuloy na ipapatupad ng otoridad para ang mga mabuting aral ay hindi maiiwan sa mga pahina

${ }^{19}$ Emerita Quito, "Philosophy of Education for Filipinos," in A Life of Philosophy: Festschrift in Honor of Emerita S. Quito (Manila: De La Salle University, 1990), 766.

(c) 2014 F.P.A. Demeterio III

http://www.kritike.org/journal/issue 14/demeterio june2014.pdf

ISSN 1908-7330

(cc) BY-NC 
ng libro at sa silid aralan. Marahil kinakailangan ng ating mga lokal at nasyunal na pamahalaan na maglaan ng pondo para ipadala ang ating mga guro, ilang opisyal at mamayahag sa mga maayos at magandang lungsod sa ibang bansa para obserbahan at aralin ang kanilang mga sinasagawang sustenableng pangangalaga sa kani-kanilang komunidad at kapaligiran.

\section{Kongklusyon}

Ang kontrobersiyal na teksto ni Brown at ang mga madamdaming reaksiyon ng mga Manilenyo at Pilipino laban o pabor nito ay hindi dapat maging isang yugto lamang sa ating kasaysayan na biglang umusbong at bigla ring nabaon sa limot. Maganda itong gawing pagkakataon para mapagnilayan kung ano ba talaga ang mga sanhi sa likod ng negatibong imahen ng Lungsod, at alin sa mga ito ang kaya nating tugunan. Kailangan nating pagandahin at ayusin ang Maynila, hindi para kay Brown o kay Danes, kung hindi para sa ating sarili, bilang mga Manilenyo, sa mga susunod pang mga Manilenyo, at pati na sa lahat ng Pilipino na may karapatan ding magkaroon ng pambansang kabisera na maaaring ipagmamalaki sa buong mundo.

Department of Filipino, De La Salle University-Manila, Philippines

\section{References}

Alcazaren, Paulo, "Eleven Resolutions for Metro Manila," in Philippine Star, (01 January 2011). Date Accessed: 02 November 2013, $<$ http://www.philstar.com/modern-living/643880/11-resolutionsmetro-manila>.

"Once upon a Fascinating Manila," in Philippine Star, (26

October 2013). Date Accessed: 02 November 2013, $<$ http://www.philstar.com/modern-living/2013/10/26/1249339/onceupon-fascinating-manila>.

"Ten Reasons Why Metro Manila is Losing its Soul," in Philippine Star, (29 June 2013). Date Accessed: 02 November 2013, $<$ http://www.philstar.com/modern-living/2013/06/29/959355/tenreasons-why-metro-manila-losing-its-soul>.

"Twety-Five Truths about Metro Manila," in Philippine Star, (24 July 2011). Date Accessed: 02 November 2013, $<$ http://www.philstar.com/sunday-life/709025/25-truths-aboutmetro-manila>.

Brown, Dan, Inferno: a Novel (New York: Doubleday, 2013). 
De Vera, Ruel, "Jessica Hagedorn on Dan Brown," in Inquirer Lifestyle, (15 July 2013). Date Accessed: 01 November 2013, $<$ http://lifestyle.inquirer.net/113993/jessica-hagedorn-on-danbrown-he-has-all-the-right-to-call-manila-gates-of-hell-but-he-cantwrite>.

Jefferson, Mark, "The Law of the Primate City," in Geographical Review, 29:2 (April 1939), 226-232.

Malone, Robert, "World's Densest Cities," in Forbes.Com, (21 December 2006). Date Accessed: 01 November 2013, <http://www.forbes.com/ 2006/12/20/worlds-most-congested-cities-biz-energycx_rm_1221congested_slide.html>.

McTavish, James, "Prostitution in the Philippines: a Time for Change," in CBCP-NASSA Caritas Filipinas Foundation, (2010). Date Accessed: 01 November 2013, <http://www.nassa.org.ph/wp-content/uploads/ 2012/08/HIV/Prostitution\%20in\%20the\%20Philippines\%20$\% 20 \mathrm{a} \% 20$ time\%20for \% 20change.pdf>.

Porcalla, Delon, "Gates of Hell? Pinoys Know Better," in Philippine Star, (25 May 2013). Date Accessed: 02 November 2013, <http://www.philstar.com/headlines/2013/05/25/946090/gates-hellpinoys-know-better $>$.

Quito, Emerita, "Philosophy of Education for Filipinos," in A Life of Philosophy: Festschrift in Honor of Emerita S. Quito (Manila: De La Salle University, 1990), 761-766.

Roderos, Ray Simon, "Reshaping Metro Manila: Gentrification, Displacement, and the Challenge Facing theUrban Capital," in Social Transformations: Journal of the Global South, 1:2 (2013), 79-103.

"The largest Cities in the World by Land Area, Population and Density," In Citymayors.Com, (06 January 2007). Date Accessed: 01 November 2013, <http://www.citymayors.com/statistics/largest-cities-density125.html>.

Tolentino, Francis, "Letter to Dan Brown," in Dinglasan, Rouchelle, "MMDA to Dan Brown: Manila is Portal to Heaven not Gates of Hell," in GMA News Online, (23 May 2013). Date Accessed: 02 November 2013, <http://www.gmanetwork.com/news/story/ 309709/news/metromanila/mmda-to-dan-brown-manila-is-portalto-heaven-not-gates-of-hell>.

Velasco, Aida, "City Innovation Systems: the Metro Manila Experience," in Policy Brief: Towards Innovative, Liveable and Prosperous Asian Cities, 3:2 (Undated), 1-4.

Wilson, James \& Kelling, George, "The Police and Neighborhood Safety: Broken Windows," in Manhattan Institute for Policy Research. Date

(C) 2014 F.P.A. Demeterio III http://www.kritike.org/journal/issue 14/demeterio june2014.pdf ISSN 1908-7330 
Accessed: 02 November 2013, <http://www.manhattaninstitute.org/pdf/_atlantic_monthly-broken_windows.pdf $>$. 